DOI: https://doi.org/10.46296/ig.v4i8edespdic.0051

\title{
CONTRIBUCIONES DEL TPM EN LA MEJORA DE LA GESTIÓN DEL MANTENIMIENTO
}

\section{CONTRIBUTIONS OF THE TPM IN THE IMPROVEMENT OF MAINTENANCE MANAGEMENT}

\author{
Solís-Meza Mario ${ }^{1}$; Torres-Rodríguez Roberto ${ }^{2}$ \\ ${ }_{1}$ Programa de Mantenimiento Industrial, Instituto de Postgrado, Universidad Técnica de \\ Manabí. Portoviejo, Ecuador. Correo: msolis5855@utm.edu.ec. \\ 2 Docente Departamento de Mecánica, Facultad de Matemáticas Físicas y Químicas, \\ Universidad Técnica de Manabí. Portoviejo, Ecuador. Correo: roberto.torres@utm.edu.ec.
}

\begin{abstract}
Resumen
El mantenimiento productivo total es una de las metodologías que garantiza alta eficacia y eficiencia en la gestión del mantenimiento industrial. El objetivo de la investigación es definir las contribuciones del mantenimiento productivo total en la mejora de la gestión del mantenimiento práctica de implementación para presentar una descripción general de las prácticas de implementación. Se revisaron 39 fuentes bibliográficas compuestas por 25 artículos científicos, 2 libros, 4 tesis doctorales y 8 artículos publicados en eventos científicos. Los resultados alcanzados a partir del análisis comparativo de la información y su discusión. Los resultados fundamental de la investigación es la contribución del TPM y mejora significativa en la gestión del mantenimiento este artículo confirma el impacto positivo que aporta el desarrollo en las organizaciones y su amplia utilización en grandes corporaciones, qué se manifiesta en un incremento de la eficacia y la eficiencia de la gestión del mantenimiento.
\end{abstract}

Palabras clave: gestión del mantenimiento, mantenimiento productivo total, beneficios.

\begin{abstract}
Total productive maintenance is one of the methodologies that guarantees high effectiveness and efficiency in the management of industrial maintenance. The objective of the research is to define the contributions of total productive maintenance in the improvement of the implementation practice maintenance management to present an overview of the implementation practices. 39 bibliographic sources were reviewed, consisting of 25 scientific articles, 2 books, 4 doctoral theses and 8 articles published in scientific events. The results achieved from the comparative analysis of the information and its discussion. The fundamental results of the research is the contribution of the TPM and significant improvement in maintenance management. This article confirms the positive impact that development has on organizations and its wide use in large corporations, which is manifested in an increase in efficiency and the efficiency of maintenance management.
\end{abstract}

Keywords: maintenance management, total productive maintenance, benefits.

Información del manuscrito:

Fecha de recepción: 04 de octubre de 2021.

Fecha de aceptación: 09 de noviembre de 2021.

Fecha de publicación: 08 de diciembre de 2021. 


\section{Introducción}

Los desafíos de la dura competencia debido a la economía globalizada, obligan a las organizaciones a innovar y mejorar tanto sus procesos, productos y métodos, estos a su vez permita elevar su desempeño operativo para cumplir con los desafíos planteados por las demandas cambiantes del mercado (García Alcaraz et al., 2012) \& (Castiillo et al., 2018) y otros. Este mejoramiento va aparejado de la innovación y el mejoramiento en el proceso de mantenimiento, de actualización de sus estrategias.

El papel del mantenimiento en los sistemas de fabricación modernos es cada vez más importante para las empresas que adoptan el mantenimiento como un negocio generador de beneficios. No es raro que los departamentos de mantenimiento y operaciones sean los más grandes, y cada uno comprende el 30 por ciento de la mano de obra total y también los costos de energía, los costos de mantenimiento pueden representar la mayor parte de cualquier presupuesto operativo. (Jain, 2015)
Esto obliga a la adopción de un sistema de mantenimiento favorable para lograr los objetivos de las empresas.

La gestión moderna del mantenimiento incluye todas aquellas actividades destinadas a determinar objetivos y prioridades de mantenimiento, las estrategias y las responsabilidades. Todo ello facilita la planificación, programación y control de la ejecución del mantenimiento, buscando siempre una mejora continua y teniendo en cuenta aspectos económicos relevantes para la organización. Una adecuada gestión del mantenimiento, teniendo en cuenta el ciclo de vida de cada activo físico, debe cumplir con los objetivos de reducir los costos globales de la actividad productiva, asegurar el buen funcionamiento de los equipos y sus funciones, disminuir al máximo los riesgos para las personas y los efectos negativos sobre el medio ambiente, generando, además, procesos y actividades que soporten los objetivos mencionados (Viveros y otros, 2013).

Impulsados por los crecientes problemas relacionados con la sostenibilidad, hoy en día los 
fabricantes globales enfrentan el desafío de crear prácticas de fabricación sostenibles. En la literatura científica existen muchos estudios que promueven la sostenibilidad del diseño de producto y la fabricación, no así en la ingeniería del mantenimiento y en su gestión. La contribución de la disciplina de mantenimiento que respalda las operaciones de fabricación sostenibles todavía se centra principalmente en extender la vida útil del equipo (Melo \& Loos, 2018).

La planta de fabricación y su mantenimiento son mucho más complejos hoy que hace unos años y definitivamente se volverá más complejo en el futuro. Las organizaciones de fabricación utilizaban diferentes enfoques de mantenimiento para mejorar la eficacia del mantenimiento La situación del sistema de mantenimiento puede mejorarse con un enfoque basado en el concepto de TPM con actividades para eliminar las pérdidas que ocurren durante la producción.

El TPM es una de las mejores herramientas para hacer que las industrias manufactureras sean competitivas y efectivas, en el campo de mantenimiento. Los fabricantes deben estar respaldadas por un mantenimiento efectivo y eficiente. (Jain, 2015)

\section{Mantenimiento}

El mantenimiento, entendido como el conjunto de actividades encaminadas a garantizar el correcto funcionamiento de las máquinas e instalaciones que conforman un proceso de producción permitiendo que éste alcance su máximo rendimiento(Olarte et al., 2010).

Una de las políticas más utilizadas en los sistemas de mantenimiento es el mantenimiento preventivo planificado (MPP), es todo el conjunto de medidas de carácter técnico y organizativo mediante las cuales se lleva a cabo el mantenimiento y la reparación de los equipos. Estas medidas son elaboradas previamente según el plan que asegura el trabajo ininterrumpido de los equipos(Ferrer-Jiménez, 2015).

Entiéndase por mantenimiento todas las actividades organizativas, técnicas y económico financieras que se realizan para lograr el estado óptimo de funcionamiento de todos 
los equipos, máquinas y estructuras que conforman la empresa.

\section{TPM}

La herramienta del TPM se extendió en Japón en 1971 por Seiichi Nakajima, teniendo su origen en el seno de una importante empresa proveedora del sector del automóvil denominada Niponndenso Co. Ltd. Esta empresa, debido a la implantación de sistemas automatizados de transferencia rápida, requería de una alta fiabilidad en sus procesos, por lo que en 1961 definió una nueva visión del mantenimiento, transfiriendo la responsabilidad del mantenimiento rutinario de los equipos, del departamento de mantenimiento, a los propietarios de los procesos y trabajadores de producción, logrando grandes resultados con su modelo a partir de 1969 (MarínGarcía \& Mateo Martínez, 2013).

TPM propone una metodología prescriptiva de implantación en la cual se detallan los pasos que las empresas deben seguir para implantar el proceso y obtener los beneficios esperados. Estudios previos realizados sobre las actividades desplegadas por parte de las organizaciones relacionadas con TPM muestran algunas diferencias significativas en cuanto al proceso de implantación del TPM y a los resultados obtenidos, atribuibles a la fase en que se encuentra la implantación misma del TPM, la labor propia de cada organización, su estilo de dirección y su cultura predominante entre otros (Villegas y Velez, 2014)

EI TPM es en la actualidad una de las principales herramientas para lograr la eficiencia y competitividad, lo que supone cumplir con especificaciones de cali-dad, tiempo y costo de la producción; generalmente se ejecuta junto con la Admi-nistración Total de la Calidad (TQM, Total Quality Management, por sus siglas en inglés), que se fundamenta en la búsqueda permanente por mejorar los rendimien $\neg$ tos de procesos y los medios de producción. Además, el TPM es altamente eficaz en aquellas empresas que cuentan con operaciones automáticas y secuenciales (empresas intensivas en el uso de maquinaria), y no requiere grandes inversiones económicas para lograr el aprovechamiento de las 
instalaciones exis $\neg$ tentes (García Alcaraz y otros, 2012).

Para algunos sistemas que requieren la ejecución continua de múltiples misiones, todo el mantenimiento las actividades deben realizarse durante las pausas de mantenimiento. Sin embargo, debido al recurso de mantenimiento restricciones, no siempre es factible reparar todos los componentes. Para resolver tales problemas se combinan las estrategias de mantenimiento tales como el mantenimiento selectivo (Zhonghao Zhao y otros, 2019)

EI TPM es una estrategia compuesta por una serie de actividades ordenadas que permiten mejoras en la competitividad de la organización industrial o de servicios. El instituto Japonés de Mantenimiento de Plantas (JIPM) define el TPM como un sistema orientado a lograr cero accidentes, cero defectos y cero pérdidas (Suzuki, 2017)

En el TPM, el trabajador no es visto como miembro del equipo de mantenimiento en la mayoría ajustes de producción. El operador está capacitado para realizar muchas de las tareas diarias de mantenimiento simple y localización de fallas. El diseño de las instalaciones es una disposición organizada y funcional de departamentos, máquinas, equipos y servicios diferentes en una industria manufacturera. Está necesario tener un diseño de planta bien desarrollado para todos los recursos disponibles de la mejor manera posible manera de obtener el mayor rendimiento (Nallusamy, 2016).

\section{Beneficios del TPM}

El TPM es un método excepcional que elimina las pérdidas relacionadas con el equipo y mejora la disponibilidad y la tasa de rendimiento y tasa de calidad (MAJUMDAR, 2017).

Para la implementación del TPM, es necesario cumplir con los doce pasos de la implementación de TPM y empuje avanzar de manera constante para realizar con éxito las ocho actividades de apoyo de TPM (Shen, 2015)

Según (Shen, 2015), la aplicación dl TPM, debe estar enmarcada en las siguientes perspectivas:

1. Perseguir la máxima eficiencia del sistema de fabricación (eficiencia general), con el objetivo de mejorar la calidad de las empresas. 
2. Bajo el marco en la escena y con material, y dirigido en todo el círculo de la vida, perseguir cero desastres, cero defectos, cero averías y evitar todas las pérdidas en

avanzar.

3. Excepto el departamento de fabricación, todos los departamentos (en desarrollo

departamento, departamento de ventas, departamento de gestión) también están involucrados.

4. Participación general de los empleados, desde el operativo estrato, y por debajo de la línea del frente.

5. Logre el objetivo de cero pérdidas mediante la repetición de pequeñas actividades grupales.

Iniciativas de TPM, sugeridas y promovidas por Japón Instituto de Mantenimiento de Plantas (JIPM), involucran un plan de implementación de ocho pilares que resulta en un aumento sustancial de la productividad laboral, reducción de los costes de mantenimiento, y reducción de paradas de producción y tiempos de inactividad. Los ocho pilares de TPM incluyen mantenimiento autónomo; mantenimiento enfocado; mantenimiento planificado; calidad mantenimiento; Educación y entrenamiento; oficina TPM; gestión del desarrollo; y seguridad, salud y medio ambiente (Jain, 2015).

TPM ayuda a establecer una relación entre el mantenimiento $y$ departamentos de producción. Cualquier parada en la línea de producción debido a la falla de equipo disminuye la tasa de producción, así como la calidad del producto. Una implementación de TPM bien concebida no solo mejora la eficiencia y eficacia del equipo, sino que también aporta mejoras apreciables en otras áreas de una planta de fabricación (Jain, 2015).

(Madanhire \& Mbohwa, 2015), realizó la implantación efectiva y gradual de un sistema de mejora continua bajo la filosófica del TPM para aumentar la confiabilidad de equipos, disminuir los desperdicios por calidad de producto que se generan debido a fallas den el proceso, involucrar y comprometer a los colaboradores con la adopción de la filosofía TPM, además que al implementar el pilar de capacitación y entrenamiento se logró 
incrementar la productividad en la empresa.

En el sector de mercados de producto de primera necesidad, el mantenimiento cumple un roll muy importante cuya actividad de apoyo a las actividades comerciales, como otras actividades, el mantenimiento debe ser eficaz, eficiente y de bajo costo. Con esta actividad de mantenimiento, la máquina y equipo de producción se puede utilizar de acuerdo con el plan y no se daña durante un cierto período de tiempo que se ha planeado alcanzar (Irwansyah et al., 2019).

EI TPM es un enfoque que involucra a los empleados desde el departamento de mantenimiento y el departamento de producción hasta la gestión de equipos. Un mantenimiento autónomo por parte de operadores y actividades de grupos pequeños en cada departamento y en cada nivel. Todas las mejoras deben estandarizarse y mantenerse porque el TPM es continuo mejoras centradas en el equipamiento y la producción (Azid et al., 2019).

En la implementación del TPM, se utilizan varias herramientas $y$ metodologías entre las que se encuentra la metodología de las 5S, que proviene de los términos japonés de los cinco elementos básicos del sistema: Seiri (selección), Seiton (sistematización), Seiso (limpieza), Seiketsu (normalización) y Shitsuke (autodisciplina), las cuales deben convertirse en una forma natural de actuar (Reyes et al., 2017).

Es el mantenimiento en estos tiempos uno de los procesos de mayor importancia para lograr la competitividad de las empresas de producción, y en este contexto el TPM, constituye una de las herramientas más adecuadas para lograr este objetivo. No obstante, es importante valorar la factibilidad de su implementación en un contexto determinado, puesto que, por ejemplo: la cultura empresarial es un aspecto importante, aspecto que debe valorarse con mucha atención.

\section{Mantenimiento Autónomo}

Cuando los directivos ejecutan el mantenimiento productivo total (TPM), normalmente hacen más hincapié en la autonomía de mantenimiento tratando de crear la cultura de compromiso del operario. Es una actividad de implementación interna dentro de TPM. El 
mantenimiento autónomo no se inventó originalmente, por lo que en cuanto a depreciar el mantenimiento, pero fue el resultado de los grupos de fabricación que deseaban mejorar y regular sus equipos.

Lo cual con lleva a que un operador sea una especie de semi-propiedad, y se le enseña para que la producción no se detenga ni siquiera cuando la máquina enfrenta fallas menores. Por lo general una avería, genera pérdidas de energía, pérdidas de velocidad se producen debido a la avería de equipos y maquinaria(Acharya et al., 2018).

\section{Materiales y métodos}

Para realizar la investigación relacionada con la gestión de mantenimiento basado en el TPM se realizó la revisión de la información del contenido de 39 publicaciones sobre TPM, extraídas de bases de datos elegidas por su reputación en publicaciones

científicas y académicas, los artículos se buscaron a través de las palabras clave "TPM", "Mantenimiento Productivo Total" y "Total Productive Maintenance". El resultado de la búsqueda ofreció una extensa relación de publicaciones: artículos de revistas de investigación, libros, conferencias y fuentes online, la pregunta que se formula para realizar el proceso de selección de datos es la siguiente: ¿Qué impacto ha generado el mantenimiento integral basado en el TPM en las industrias? El resultado alcanzado se obtiene a partir del análisis comparativo de los puntos de interés y la discusión de diversos autores, se consideró publicaciones académicas, como tesis de posgrado, de diversas instituciones de educación superior y extranjeras, artículos científicos de actividades y estudio recientes, así como artículos publicados en eventos científicos.

Tabla 1. Fuentes bibliográficas consultadas.

\begin{tabular}{|c|c|c|c|c|}
\hline \multirow[t]{2}{*}{ FUENTES BIBLIOGRAFICAS } & \multicolumn{2}{|c|}{$\begin{array}{c}\text { FUENTE BIBLIOGRAFICA ULTIMOS } 10 \\
\text { AÑOS }\end{array}$} & \multicolumn{2}{|c|}{$\begin{array}{c}\text { FUENTE BIBLIOGRAFICA ULTIMOS } 5 \\
\text { AÑOS }\end{array}$} \\
\hline & UNIDADES & $\%$ & UNIDADES & $\%$ \\
\hline ARTICULO CIENTIFICO & 13 & $72 \%$ & 12 & $57 \%$ \\
\hline LIBROS & 0 & $0 \%$ & 2 & $9 \%$ \\
\hline TESIS & 2 & $11 \%$ & 2 & $10 \%$ \\
\hline CONFERENCIA & 3 & $17 \%$ & 5 & $24 \%$ \\
\hline
\end{tabular}


El resultado que reflejan la investigación de los trabajos bibliográficos de la gestión de mantenimiento basado en el TPM se pudo evidenciar la fundamentación y la efectividad de la investigación y responder a las necesidades de realización de los trabajos con mayor efectividad cuyo beneficio $y$ respuesta inmediata en el área de mantenimiento es ideal para el sector industrial.

\section{Resultados y discusión}

Los resultados obtenidos dan muestra de que la contribución del TPM a la mejora en la gestión del mantenimiento es de gran relevancia, siguiendo la lógica de presentar de cada autor los objetivos planteados definidos y las herramientas utilizadas, los beneficios y las conclusiones de la implementación del mantenimiento total productivo.

El desarrollo de la investigación se obtuvo que dentro de los resultados, en la productividad fue uno de los beneficios operacionales que destacas un mayor porcentaje de participación obtenido por la implementación del TPM y la mejora en gestión de mantenimiento, la importancia de mencionar la filosofía de los 8 pilares del TPM, 5s, el mantenimiento autónomo, mantenimiento planificado y la gestión temprana de mantenimiento fueron los más utilizados en la implementación del TPM por los autores.

A continuación, se destacan los resultados de varias investigaciones desarrolladas en la aplicación del TPM en varias empresas.

1. Autor: (Aranguren Medina, 2015)

Objetivo: Clasificar los distintos obstáculos y problemas presentados durante las diferentes etapas de implantación de TPM

Herramientas TPM: Aplicación de la filosofía de las 5s, utilizaciones de los 8 pilares del TPM, Se promueve el mantenimiento autónomo

\section{Beneficio de la aplicación del} TPM: Cabe destacar que a pesar de la resistencia de adoptar la filosofía del TPM, su beneficio permite mejorar la eficiencia de los equipos y el personal como los directivos que lo conforman

Conclusiones: Las empresas investigadas desarrollan un interés 
en común para consolidar entre ellas y poder explorar y aprender juntos, tener un mayor acceso a información, documentación respecto a la implementación del TPM

2. Autor: (Kwaso, 2017)

Objetivo: Evaluación del impacto del mantenimiento productivo total elementos en un proceso de fabricación

Herramientas TPM: La filosofía de las $5 \mathrm{~s}$, Utilizaciones de los 8 pilares del TPM, Efectividad general del equipo

Beneficio de la aplicación del TPM: El TPM no es sólo una estrategia de mantenimiento, pero también una herramienta de mejora empresarial que las empresas utilizan para permanecer competitivo mientras mejora la satisfacción y confiabilidad del cliente

La implementación de las herramientas del TPM indica la capacidad de los pilares de TPM para mejorar el negocio, sustentando estos elementos como cruciales para una implementación exitosa.

Conclusiones: TPM producen resultados positivos cuando se implementan correctamente en los procesos de fabricación. Pilar de mantenimiento autónomo y mejora focalizada. Estos autores informaron cero quejas de clientes y cero accidentes. De manera similar, los resultados de este estudio de caso muestran la implementación de las prácticas fundamentales de TPM liderando y gestión del cambio, medio ambiente, salud y seguridad, trabajo en equipo, 5S, gestión visual, mejora focalizada y el mantenimiento autónomo como pilar es una consecuencia de una OEE constante y mejorada que tiene el valor más bajo en $81,5 \%$ después de la implementación y el más alto en $93,3 \%$, en comparación con antes de la implementación con el valor OEE más bajo en $64,6 \%$ y el más alto en $89,9 \%$.

3. Autor: (Brodny \& Tutak, 2017)

Objetivo: Aplicación de elementos de la estrategia TPM para la operación Análisis de la máquina minera

Herramientas TPM: TPM, Efectividad general del equipo (OEE) Beneficio de la aplicación del TPM: Permite el análisis el cual se basó en los datos registrados por el 
sistema de automatización industrial utilizado en las minas. Método de adquisición de datos aseguró su alta credibilidad y una sincronización a tiempo completa.

Conclusiones: El análisis del uso del OEE para evaluar la eficiencia de uso y mantenimientos de equipos de minería fuente importante de información para que los servicios de mantenimiento mejoran el uso de los equipos.

4. Autor: (Chukwutoo \& Paschal, 2018)

Objetivo: Optimización del sistema de producción: una aplicación del mantenimiento productivo total (TPM)

Herramientas TPM: Sistema de producción; TPM; eficacia general del equipo; disponibilidad de equipos; tasa de rendimiento; tasa de calidad; Principio de Pareto

Beneficio de la aplicación del TPM: Creo un mayor nivel de confianza entre los empleados. Mantiene el área de trabajo limpio, ordenado, ordenado. Se promueve el buen comportamiento laboral favorable de los trabajadores.
Conclusiones: La evaluación de TPM debe ser consistente práctica para optimizar en función operativa y en consecuencia OEE ¿Se justifica la evaluación del desempeño? métodos en las industrias manufactureras eficacia y rendimiento de los, representa una fuerte reducción de todos los factores de tiempo de inactividad de 2012 a 2013 y la tasa OEE ha apreciado en silencio de 2012 a 2013 respectivamente.

El grupo de gestión y mantenimiento siempre debe apuntar a la implementación total de los ocho pilares de TPM para llevar el valor de OEE al estándar de clase mundial de $85 \%$.

5. Autor: (Díaz-Reza et al., 2018)

Objetivo: El papel del compromiso gerencial y el TPM Estrategias de implementación en beneficios de productividad

Herramientas $\quad$ TPM: $\quad$ TPM; implementación; compromiso gerencial

Beneficio de la aplicación del TPM: Desarrolló del compromiso por parte de la gerencia, el éxito de TPM y los programas de pm. Como consecuencia, los operadores TPM 
siempre deben exigir apoyo gerencial antes de iniciar cualquier programa de mantenimiento, porque el compromiso gerencial por sí solo no garantiza los beneficios

Conclusiones: estadísticamente, tres variables latentes explican el $38,3 \%$ de la variable latente de beneficios de productividad: Implementación de TPM (16,4\%), compromiso gerencial $(16,2 \%)$ e implementación de PM (5,7\%). Tales estimaciones implican que los gerentes deben prestar mucha atención a las dos primeras variables, ya que tienen los mayores efectos. Aunque el impacto directo de la implementación de PM es bajo en beneficios de productividad, el efecto indirecto tiene un valor de 0,097, que puede explicar el 4,55\%. En el que finalmente, los efectos totales de la implementación de PM sobre los beneficios de productividad tienen un valor de 0.218 unidades, y esta variable latente explica hasta el $10,2 \%$. En otras palabras, el mantenimiento preventivo como parte de la implementación de TPM es vital si las empresas buscan obtener beneficios de productividad.
6. Autor: (Fam et al., 2018)

Objetivo: Estudiar la efectividad de lean técnicas de fabricación para reducir el desperdicio y aumentar la OEE en industria de fabricación de papel y productos de papel.

Herramientas TPM: Lean Team, diagrama de Ishikawa, Mapeo de flujo de valor (VSM), Trabajo estándar (SW), Kaizen, OEE

Beneficio de la aplicación del TPM: Los resultados empíricos proporcionan varios puntos de vista valiosos para gerentes para mejorar la gestión de la calidad y la productividad de su proceso de producción. Posteriormente, las organizaciones relacionadas pueden responder a la regulación, la política y el control haciendo esfuerzos para reducir el tiempo de fraguado de la máquina y el tiempo de inactividad de la máquina, y evitar de comprar papeles de mala calidad. El Kaizen también debe ser sostenido en la industria de fabricación de papel y productos de papel.

Conclusiones: El modelo de regresión cumple con el Durbin Watson requisito donde el valor de Durbin Watson es igual a 1,723, que cae en el rango de 1,5 a 2,5. 
Además, el modelo es capaz de explicar $74,7 \%$ de la variación total en la OEE.

7. Autor: (Adesta et al., 2018)

Objetivo: Evaluación de 8 pilares del Mantenimiento Productivo Total (TPM) implementación y su contribución al rendimiento de fabricación

Herramientas TPM: Tpm y sus 8 pilares

Beneficio de la aplicación del TPM: Mejora los planes de mantenimientos el uso de los pilares ya que crea un cambio y compromiso por parte de los operarios como la gerencia.

La implementación de 8 pilares de TPM en Indonesia Industrias manufacturera fue relativamente bueno. Donde los 4 pilares, a saber: Mantenimiento Autónomo, la mejora continua, el mantenimiento de la calidad y la educación y la formación funcionan muy bien. Los otros 4 pilares, a saber: mantenimiento planificado, seguridad-salud y medio ambiente que permite el mejor desarrollo de la empresa.

Conclusiones: Las variables insignificantes en el desempeño de la manufactura también deben revisarse mediante cuestionarios relacionados. Desde el modelo estructural, también puede ser concluyó que el modelo de los pilares tpm y la relación $\mathrm{mp}$ es confiable, positivo y fuerte, como tpm los pilares pueden explicar el $62,6 \%$ de variabilidad de $\mathrm{mp}$, mientras que otros factores influyeron en el resto $(37,4 \%)$

8. Autor: (Anaya Vega, 2020)

Objetivo: Diseñar la propuesta de implementación de la metodología de Mantenimiento Productivo Total (TPM), en la Empresa Colombiana de Cementos S.A.S. del sector construcción ubicada en la región de Rio Claro - Antioquia

Herramientas TPM: Mejora Continua, Mantenimiento Productivo Total, Negocios Desarrollo, 5S, Lean Manufacturing, Six Sigma, Análisis de modo y efecto de falla

Beneficio de la aplicación del TPM: El diseño de implementación de TPM Se ajusta a los programas de capacitación, formación y desarrollo de habilidades blandas al personal de mantenimiento, cuya técnica le permiten el cumplimiento de los objetivos 
Conclusiones: Implementar el tpm a la empresa de cemento, permitieron una Tasa Interna de Retorno del $28 \%$, siendo una gran oportunidad de tipo financiera demostrado en el valor de ahorro por implementación de la metodología.

9. Autor: (Navia Núñez, 2020)

Objetivo: Impacto de la integración de ISO 9001 y TPM

Herramientas TPM: Mantenimiento Productivo Total, ISO 9001, Sistemas Integrados de Gestión, Sistemas de gestión.

\section{Beneficio de la aplicación del} TPM: Los beneficios tácitos expresados por los gerentes fueron la creación de un sentido de propiedad entre los operadores a través de la interacción de la cláusula de "Soporte" en base al cumplimiento de la subcláusula "Competencia" con los pilares TPM de Mantenimiento Autónomo y Educación y Formación, como se muestra en el modelo. Esto se logró a través de la educación de los empleados, la capacitación y la creación de la polivalencia de los empleados.
Conclusiones: La integración del Mantenimiento Productivo Total (TPM) y tres diferentes estándares de sistemas de gestión específicos de la función, a saber, ISO 9001: 2015, ISO 45001: 2018 e ISO 14001: 2015. La integración desarrolla en los empleados en la organización a la creación de una cultura organizacional que se involucre en un ciclo continuo de autoevaluación, corrección y mejora de operación

10. Autor: (Palomino-Valles et al., 2020)

Objetivo: Estudio de mantenimiento centrado en TPM y mantenimiento RCM. Su enfoque es basado en los primeros pilares del TPM

Herramientas TPM: Modelo de gestión de mantenimiento, TPM, $\mathrm{RCM}$

Beneficio de la aplicación del TPM: Modelos integrados, las mejores metodologías para el desempeño de la organización. Esto permite un mejor control sobre las actividades de mantenimiento realizadas y ayuda a determinar las componentes críticas.

Conclusiones: Con la propuesta de mejora, es posible un aumento del 
$5 \%$ en la disponibilidad general de la flota de maquinaria pesada y minicargadoras, lo que permitiría a la empresa en estudio estar en niveles competitivos dentro del mercado nacional de alquiler de maquinaria en el sector de la construcción. Simulando el proceso de mantenimiento propuesto, el tiempo promedio de espera entre fallas se puede reducir de 13 horas a 7 horas. Esto significa una reducción del 15\% en el tiempo de inactividad.

11. Autor: (Dobra \& Jósvai, 2021)

Objetivo: El impacto del desempeño humano en la OEE en la adopción de Nuevas tecnologías de producción

Herramientas: TPM: OEE.

Beneficio de la aplicación del TPM: Formación de conciencia del operador, con un enfoque principal en el tiempo de falla debido a errores del usuario, van generando tiempos de inactividad. Período de capacitación del operador debido a la posibilidad de evaluar las ineficiencias de los procesos humanos con el marco propuesto

Permite la evaluación del rendimiento de la maquinaria en un y entorno interconectado al distinguir entre humanos y tecnológicos.
Atreves de este estudio se propone un marco integral para evaluar el desempeño de la tecnología de producción.

Conclusiones: El propósito de este análisis para mejorar el rendimiento del sistema y aumentar el valor OEE. Utilizando combinación de agrupamiento como método de minería de datos y el análisis humano con balanceo de líneas dio como resultado un rendimiento del $66 \%$ y mejora del valor OEE del $11 \%$ en la práctica. el presentado la técnica de análisis se puede utilizar con una inversión mínima, no solo en líneas de montaje, pero también en máquinas individuales donde hay muchos datos disponibles y el tiempo de ciclo o takt time debe reducirse para obtener mayores rendimientos o un valor OEE más alto.

\section{Implementación de las $5 \mathrm{~s}$}

La implementación de la metodología $5 S$ se pudo resaltar los resultados que se obtiene en la mayoría de las organizaciones han mostrado un compromiso total con la filosofía, para la mejora continua de la calidad y productividad en las plazas laborales y la importancia de la $5 S$ que proviene de los términos 
japonés de los cinco elementos básicos del sistema: Seiri (selección), Seiton (sistematización), Seiso (limpieza), Seiketsu (normalización) y Shitsuke (autodisciplina) .La implementación de 5S en todos los niveles ha facilitado que las organizaciones de fabricación acumulen beneficios significativos, como la mejora en la organización general, la productividad, la calidad, la seguridad, los valores de la moral de los empleados, la utilización eficaz del espacio de trabajo y la optimización de costos. Los resultados también muestran la eliminación de problemas crónicos graves del sistema de producción, como retrasos, averías, empleados desmoralizados, disminución de las ganancias y clientes insatisfechos a través de la implementación integral de las 5S (Ho, 1997),(Hubbard, 1999), (Chapman, 2005), (Sorooshian et al., 2012), (Singh \& Ahuja, 2014), (Jiménez et al., 2015), (Veres et al., 2018).
Contribución para mejora de la gestión de mantenimiento con la implementación de las $5 \mathrm{~s}$

El tipo de investigación, en el cual permitió concluir en las reflexiones de diferentes autores en el que han adoptado la filosofía de las $5 \mathrm{~s}$, les permite mejorar la eficiencia de los equipos y personal como los gerentes que la integran mejorar la gestión de mantenimiento atreves de estrategia, sino también una herramienta de mejora empresarial que ayuda a las empresas a mantenerse competitivas mientras mejora la satisfacción del cliente y la confiabilidad al mismo tiempo. La implementación de las herramientas TPM demuestra la capacidad de los pilares para mejorar el negocio y respalda estos elementos como críticos para el éxito, en el diseño de implementación de TPM que corresponde a programas de capacitación y desarrollo de habilidades para el personal de mantenimiento, dé tal manera les permite alcanzar las metas con la mejora en la gestión de mantenimiento(Aranguren Medina, 2015) (Kwaso, 2017) (Anaya Vega, 2020). 


\section{Conclusiones}

El mantenimiento integral basado en

el TPM basado en las distintas investigaciones expuestas tiene como objetivos en desarrollar fundamentalmente TPM, definiéndose las relaciones jerárquicas que los regulan y sus semejanzas con los facilitadores propios de la innovación continua a través de la valoración del impacto que pueden tener sobre sus objetivos de desarrollo y la relación de jerarquías existente entre los investigadores.

La filosofía basada TPM busca la mejora de la tasa de desempeño del uso del equipo, la evaluación de los precios totales del equipo conforme el tiempo y la incidencia de las intervenciones en el costo de mantenimiento durante su ciclo de vida, el nivel de intervenciones y en especial la operativa. Además, tiene como fin participar de cada una de las áreas en la investigación de mejoras de productividad, por lo cual involucra cambios

en comportamiento. $Y$ crea un ámbito más atractivo para laborar en el cual cada miembro tiene papel importante dentro de la empresa.
Los resultados mostraron que la metodología del TPM establece ventajas que integran el desarrollo de procesos por reducción de costos mejor uso del área de trabajo, prevención de pérdida de herramientas, incremento del proceso, crecimiento de la eficiencia, acortamiento del tiempo primordial para buscar cosas elementales, trabajo improvisado condiciones para los trabajadores, Reducción del precio de mantenimiento de la máquina entre otros beneficios (Aruquipa Nina \& Paz Zeballos).

\section{Bibliografía}

Acharya, A., Garg, D., Singh, N., \& Gahlaut, U. (2018). Plant effectiveness improvement of overall equipment effectiveness using autonomous maintenance training:-A case study. Int. J. Mech. Prod. Eng. Res. Dev, 9, 103-112.

Adesta, E., Prabowo, H., \& Agusman, D. (2018). Evaluating 8 pillars of Total Productive Maintenance (TPM) implementation and their contribution to manufacturing performance. IOP Conference Series: Materials Science and Engineering, 
Anaya Vega, G. G. (2020). Diseño de la propuesta de implementación de un sistema de mantenimiento productivo total TPM para la Empresa Colombiana de Cementos SAS en la región de Rio Claro-Antioquia Universidad EAN].

Aranguren Medina, J. A. (2015). Implantación exitosa de TPM en la industria colombiana Universidad EAFIT].

Aruquipa Nina, D. F., \& Paz Zeballos, R. Plan de mantenimiento productivo total (TPM) en el Centro de Mantenimiento de la FELCN Aranjuez, zona Sur de la ciudad de La Pazz

Azid, N., Shamsudin, S., Yusoff, M., \& Samat, H. (2019). Conceptual Analysis and Survey of Total Productive Maintenance (TPM) and Reliability Centered Maintenance (RCM) Relationship. IOP Conference Series: Materials Science and Engineering,

Brodny, J., \& Tutak, M. (2017). Application of elements of TPM strategy for operation analysis of mining machine. IOP conference series: earth and environmental science,

Castiillo, Á., Fernández, L., \& Ángeles, L. (2018). Immpacto de TPM en el desempeño operativo de las Empresas Industriales del Sur de
Tamaulipas.

Ingenieria Industrial.

Chapman, C. D. (2005). Clean house with lean $5 \mathrm{~S}$. Quality progress, 38(6), 27-32.

Chukwutoo, C. I., \& Paschal, S. E. (2018). Optimization of Production System: An Application of Total Productive Maintenance (TPM). Archives of Current Research International, 1-9.

Díaz-Reza, J. R., García-Alcaraz, J. L., Avelar-Sosa, L., MendozaFong, J. R., Sáenz Diez-Muro, J. C., \& Blanco-Fernández, J. (2018). The role of managerial commitment and TPM implementation strategies in productivity benefits. Applied Sciences, 8(7), 1153.

Dobra, P., \& Jósvai, J. (2021). Enhance of OEE by hybrid analysis at the automotive semi-automatic assembly lines.

Procedia Manufacturing, 54, 184-190.

Emplean encuestas, sondeos y ese tipo de herramientas. Es lo que ocurre con los estudios antropológicos, estadísticos o las exploraciones de intención de voto. Fuente: https://concepto.de/tipos-deinvestigacion/\#ixzz6vliOndDk

Fam, S., Ismail, N., Yanto, H., Prastyo, D., \& Lau, B. (2018). Lean manufacturing and overall equipment efficiency (OEE) in paper manufacturing 
and paper products industry. Journal of Advanced Manufacturing Technology (JAMT), $12(1$ (2)), 461-474.

Ferrer-Jiménez, B. (2015). Mantenimiento preventivo en reductor de velocidad de grúas indias de extracción de mineral. Ciencia \& Futuro, 5(1), 68-89.

García Alcaraz, J. L., Romero González, J., \& Noriega Morales, S. A. (2012). El éxito del mantenimiento productivo total y su relación con los factores administrativos. Contaduría y administración, 57(4), 173-196.

Ho, S. K. (1997). Workplace learning: the 5-S way. Journal of Workplace Learning.

Hubbard, R. (1999). Case study on the $5 \mathrm{~S}$ program: the five pillars of the visual workplace. Hospital materiel management quarterly, 20(4), 24-28.

Irwansyah, D., Harahap, M. R. F., Erliana, C. I., Abdullah, D., Sari, A., Siregar, N., AchmadDaengs, G., Indahingwati, A., Sumartono, E., \& Wilujeng, S. (2019). Improvement Suggestion Performance of Blowing Machine Line 4 with Total Productive Maintenance (TPM) Method at PT. CocaCola Amatil Indonesia MedanUnit. Journal of Physics: Conference Series,
Jain, A., Bhatti, R. S, and Singh, H. (2015) OEE enhancement in SMEs through mobile maintenance: A TPM concept. International Journal of Quality \& Reliability Management. DOI: 10.1108/IJQRM-05-20130088

Jiménez, M., Romero, L., Domínguez, M., \& del Mar Espinosa, M. (2015). 5S methodology implementation in the laboratories of an industrial engineering university school. Safety science, 78, 163-172.

Kwaso, M. J. (2017). Evaluating the Impact of TPM (Total Productive Maintenance) Elements on a Manufacturing Process. University of Johannesburg (South Africa).

Madanhire, I., \& Mbohwa, C. (2015). Implementing successful Total Productive Maintenance (TPM) in a manufacturing plant. Proceedings of the world congress on engineering,

MAJUMDAR, S. N. G. (2017). Enhancement of overall equipment effectiveness using total productive maintenance in a manufacturing industry. International Journal of Performability Engineering, 13(2), 173-188.

Marín-García, J. A., \& Mateo Martínez, R. (2013). Barreras 
y facilitadores de la implantación del TPM. Intangible Capital, 9(3), 823853.

Melo, F. T., \& Loos, M. J. (2018). Análise da metodologia da Manutenção Produtiva Total (TPM): Estudo de caso. Revista Espacios, 39(3).

Nallusamy, S. (2016). Enhancement of productivity and efficiency of CNC machines in a small scale industry using total productive maintenance. International Journal of Engineering Research in Africa,

Navia Núñez, J. F. (2020). Impact of ISO 9001 and TPM Integration.

Olarte, W., Botero, M., \& Cañon, B. (2010). Importancia del mantenimiento industrial dentro de los procesos de producción. Scientia et technica, 1(44), 354-356.

Palomino-Valles, A., TokumoriWong, M., Castro-Rangel, P., Raymundo-lbañez, C., \& Dominguez, F. (2020). TPM Maintenance Management Model Focused on Reliability that Enables the Increase of the Availability of Heavy Equipment in the Construction Sector. IOP Conference Series: Materials Science and Engineering,

Reyes, J. V., Aguilar-Sánchez, L. A., Hernández-Valencia, J. L., \&
Mejías-Acosta, A. (2017). La Metodología 5S como estrategia para la mejora continua en industrias del Ecuador y su impacto en la Seguridad y Salud Laboral. Polo del conocimiento, 2(7), 1040-1059.

Shen, C.-C. (2015). Discussion on key successful factors of TPM in enterprises. Journal of applied research and technology, 13(3), 425-427.

Singh, A., \& Ahuja, I. S. (2014). Evaluating the impact of $5 S$ methodology on manufacturing performance. International Journal of Business Continuity and Risk Management, 5(4), 272-305.

Sorooshian, S., Salimi, M., Bavani, S., \& Aminattaheri, H. (2012). Case report: Experience of $5 S$ implementation. Journal of Applied Sciences Research, 8(7), 3855-3859.

Suzuki, T. (2017). TPM en industrias de proceso. Routledge.

Veres, C., Marian, L., Moica, S., \& AlAkel, K. (2018). Case study concerning $5 \mathrm{~S}$ method impact in an automotive company. Procedia Manufacturing, 22, 900-905.

Villegas, G. y Vélez, A. (2014) Implantación del Mantenimiento Productivo Total - TPM - en escenarios de fusión corporativa: Resultados de una 
investigación. 12th Latin

American and Caribbean

Conference for Engineering

and Technology "Excellence

in Engineering to Enhance a Country's Productivity". July 22 to 24, 2014 Guayaquil, Ecuador

Viveros, $\quad$ P., $\quad$ Stegmaier,R., Kristjanpoller, F. Barbera, L.Crespo, A. (2013) Propuesta de un modelo de gestión de mantenimiento y sus principales herramientas de apoyo. Ingeniare. Revista chilena de ingeniería, vol. 21(1).

Zhonghao Zhao, Boping Xiao, Naichao Wang, Xiaoyuan Yan and Lin Ma (2019) Selective Maintenance Optimization for a Multi-State System Considering Human Reliability. Symmetry 11, 652; doi:10.3390/sym11050652. 